\title{
The Future of in Vitro Fertilisation: Bright or A Little Dulled?
}

\author{
P Hollands* \\ Freelance Consultant Clinical Scientist, UK
}

Submission: February 01, 2021; Published: February 04, 2021

*Corresponding author: P Hollands, Freelance Consultant Clinical Scientist, UK

\section{Opinion}

In the early 1980's I was working with Bob Edwards and Patrick Steptoe at Bourn Hall Clinic as one of the first ever Clinical Embryologists in the world. These were pioneering times because the treatment was still very new and there was still much to learn about male and female infertility and how to best handle human embryos in the laboratory. The overall live birth rate in those early days was around $30 \%$ when considering all age groups and all diagnoses. This overall live birth rate has not changed since these early days despite the efforts to improve it and despite the fact that some clinics make unsupported claims of high live birth rates. The laboratory technology at Bourn Hall was entirely manual and all of the reagents and culture media were prepared at Bourn Hall using starting materials purchased largely from scientific suppliers. The treatment was on a partially inpatient basis with the female patient staying at Bourn Hall for up to 3 weeks during treatment and the male patient living either locally in the village of Bourn or in nearby Cambridge. At this time, it was already possible to freeze human embryos and frozen donor sperm was available if the male had insufficient sperm for in vitro fertilisation. It is easy to look back with rose tinted glasses but these early days of IVF in my opinion were the best.

In the late 1980's the regulation of IVF began to develop in the UK firstly with the Interim Licensing Authority which then blossomed into the Human Fertilisation and Embryology Authority (HFEA). There are now regulatory organizations in most Countries which ensure the safety of patients and the efficacy of treatment. This high level of regulation is critical in assisted reproduction.

The process of IVF is now on a totally outpatient basis. Female patients visit the clinic for their monitoring scans and they administer their own medication by injection. The egg collection is carried out under light sedation and the patient goes home later that day. She then returns for the embryo replacement and carries out a home pregnancy test when the time comes to see if the treatment worked. This places a considerable additional burden on the female patients which we would all do well to carefully think about. The role of the male is even less in that he is only needed to produce a semen sample on the day of egg collection. It is even possible to freeze semen before the treatment cycle and I have been involved in many IVF treatments where the male partner was overseas at the time of the treatment. This detachment of the male and female patient in the process and the demands put onto the female patient in particular does nothing for the overall success of the treatment. Everyone agrees that a stressed patient means a patient who is less likely to succeed and the delivery of modern IVF means that stress levels are potentially high for all patients. In addition, there are patients who attend fertility clinics describing 'infertility' who are immediately placed on an IVF treatment cycle. No consideration is taken of the general and mental health of the patient or whether or not simple interventions such as weight loss or life-style changes may significantly increase their chances of achieving a natural pregnancy. The drive to go for a high profit IVF cycle is irresistible and this is another area for some serious soul searching by those running IVF clinics.

Moving forward in embryology the next big innovation in IVF was Intracytoplasmic Sperm Injection (ICSI). This was originally developed to use for male patients who did not produce enough sperm for IVF and for those patients who had enough sperm for IVF but still suffered failed fertilisation. In this context ICSI was a great innovation. The drawbacks were that it involves considerable manipulation of human gametes, it requires additional training for clinical embryologists to become competent in the technique and the equipment required for the microinjection is expensive. As a result, when ICSI was offered it involved a significant extra cost to patients. Today ICSI is the most overused technique in modern IVF. Many clinics boast that they use ICSI in almost all patients and patients see this as a good thing because of the way in which it is sold to them as a 'guarantee' of fertilisation. The means that 
patients who have no clinical need for ICSI still undergo ICSI at considerable additional cost. The fertilisation 'guarantee' is also false marketing as it is well known that failed fertilisation is still possible despite using ICSI. The use of medical technology which is not clinically indicated is not allowed in all other areas of clinical practice so why does this happen in IVF? This is like telling someone with an in growing toe-nail that they need a leg amputation!

The problems in IVF revolve around the development of IVF as a profit-making procedure. There is nothing inherently wrong with this as there are many other private clinics and hospitals offering other treatments for profit. Nevertheless, in IVF there are now thousands of clinics worldwide and some of these are 'mega' clinics who treat thousands of patients per annum and generate a massive income. This income is enhanced considerably in all clinics by optimizing the use of ICSI even when it is not indicated. This is unethical and an exploitation of vulnerable patients who trust the advice they get from clinics.

Following on from the over-use of ICSI there are currently many more so called 'add-ons' which are offered to patients to allegedly increase their overall chances of a live birth. There are too many of these from 'embryo glue' to 'endometrial scratching' but the problem is that none of these 'add-ons' have been proven to be effective so once again we have IVF clinics exploiting the hopes and fears of fertility patients. Perhaps the most ridiculous and expensive of these 'add-ons' is technology which involves creating a time-lapse video of embryonic development and then claiming that this video will enable the clinical embryologist to select the 'best' embryo to replace into the mother. The equipment needed to create such a video is extremely expensive and the additional fee to the patients is high. The overall benefit of this technology to live birth rate is zero. This use of 'add-ons' in IVF is a scandal ready to break and when it does there may be many lawsuits from angry patients who have paid money for pointless procedures and technology.

In my opinion IVF today has stagnated with no real increase in live birth rate but ever-increasing profits for clinics. Part of the reason for this may be that some clinics are owned by large corporations and others are run independently by profit focussed businessmen with little thought for fertility patients. Patient feedback often reflects this lack of compassion by clinics and some patients are beginning to question the ever-increasing cost of treatment. The only way forward from now on is a complete change in mind-set of those people running IVF clinics to return to ethical, honest practice where nothing is offered unless it has a proven benefit to the patient. All healthcare professionals have a duty of care to their patients which means they should only do things which will benefit the patient. At present in IVF the only people benefitting are the clinics. This is not the legacy which Edwards and Steptoe left us and it is time that someone spoke out to protect their legacy and to protect patients.

Despite these dire current problems in IVF there is an interesting future for IVF. The first thing which needs to happen is a complete revision of the technology used in the embryology laboratory to move away from manual, error prone, procedures to true automation. This is not a time-lapse video of a developing embryo. This is technology which can bring together egg and sperm, allow fertilisation, properly monitor this process by assessing the quality and components in the culture media and perhaps using artificial intelligence to decide which are the embryos most likely to form a pregnancy. In addition, we need much more understanding of the process of implantation and how to optimise it to optimise live birth rate. Developing new technology for the processing and handling of sperm is needed. This technology has not really changed since 1978 and it a glaring problem to anyone with even a basic understanding on IVF. This will not mean some sort of automated ICSI. It will mean a new level of understanding on both the physiology and pathology of sperm and a considerable change in the technology used to prepare sperm for IVF.

These and many other developments may bring IVF into the 21st Century instead of wallowing in the 20th Century. It is also likely that even with perfect embryology, andrology and clinical practice that the success rate of IVF may still not increase. This could be because the success rate we see is that which is defined by nature and no human intervention can change it. If this proves to be true, then patients will have to accept that a $70 \%$ failure rate of IVF is to be expected because even the most brilliant minds cannot change things which are inevitable in nature.

In summary, I believe that the practice of IVF today is at an all-time low because of the factors I have described above. Those providing IVF need to think carefully about the service they are offering and whether or not this service is always in the best interests of their patients. Research scientists need to receive the funding and facilities needed to develop new, safe and effective technology for IVF. This needs collaboration between Universities and private clinics and Government support. Edwards and Steptoe left us an amazing legacy, my plea is that we do not ruin this legacy by greed, unethical practice and a complete blindness to the future. 

(CC) This work is licensed under Creative DOI: 10.19080/JGWH.2021.20.556049
Your next submission with Juniper Publishers will reach you the below assets

- Quality Editorial service

- Swift Peer Review

- Reprints availability

- E-prints Service

- Manuscript Podcast for convenient understanding

- Global attainment for your research

- Manuscript accessibility in different formats

( Pdf, E-pub, Full Tsext, Audio)

- Unceasing customer service

Track the below URL for one-step submission https://juniperpublishers.com/online-submission.php 